\title{
VERBAL FLUENCIES ASSOCIATED FACTORS IN ELDERLY
}

L. Caldas, H. Espirito-Santo, J. Matreno, M. Marques, I.T. Pena, M.D. Costa, A. Costa, D. Simões, A. Conde, A.R. Correia, R. Almeida, S. Moitinho, F. Rodrigues, S.C. Simões, L. Lemos, F. Daniel

Psychology, Instituto Superior Miguel Torga, Coimbra, Portugal

Verbal fluency (VF) involves complex processes and has been a good marker of cognitive decline. However, the literature is inconsistent concerning to witch factors are associated with VF.

Our aims are to analyze the relationship between both phonemic verbal fluency (PVF) and semantic verbal fluency (SVF) and sociodemographic and psychopathological variables, and explore which emerge as significant predictors.

A subsample of 429 of healthy institutionalized elderly from the Aging Trajectories at Coimbra Council Project were surveyed (60 to 100 years; mean age $=80.38 \pm 7.24)$, the majority was women $(76.9 \%)$, without a partner $(82.2 \%)$, without education or with less than four years of education (85.7\%), manual occupation (90.1\%), and attending day care centers. We evaluated VF phonetically (letters $\mathrm{P}, \mathrm{M}, \mathrm{R}$ ) and semantically (animals and food), anxiety symptoms through the Geriatric Anxiety Inventory (GAI), depressive symptoms through Geriatric Depression Scale (GDS), and feelings of loneliness through Loneliness Scale (UCLA).

PVF was significantly related with education, occupation, GAI, and GDS. SVF was significantly associated with age, education, occupation, and GDS. Furthermore, SVF scores were worse in elderly men and in those living in night care center, and PVF scores were lower in those with high levels of anxiety symptomatology. In logistic regression analysis none of the variables accounted for the variance in PVF. The only predictor of SVF was sex.

In conclusion, this study allowed us to elucidate the only key factor underlying verbal fluency. Being a man may affect SVF performance in institutionalized elderly. 